\title{
Trends in Hospital Admissions Associated with an Acute Kidney Injury in England 1998-2020: a Repeated Cross-Sectional Study
}

\author{
Zuzanna Bien $^{1,2}$ - Alexander J. Fowler ${ }^{1,2} \cdot$ Alexander J. Robbins $^{1,2} \cdot$ Rupert M. Pearse $^{1,2} \cdot$ John R. Prowle ${ }^{1,2}$. \\ Yize I. Wan ${ }^{1,2}$
}

Accepted: 17 January 2022 / Published online: 10 February 2022

(c) The Author(s) 2022

\begin{abstract}
A rapid rise in diagnosis of acute kidney injury (AKI) has been observed in the UK and worldwide. It is unclear whether this reflects true growth in incidence or improved detection. To investigate this, aggregate data from Hospital Episode Statistics database on all admissions to National Health Service (NHS) hospitals in England 1998/1999 to 2019/2020 were used to extract the number of AKI diagnoses in different age groups and compare them to diseases of similar incidence (pneumonia, heart failure, dementia) based on ICD-10 coding. Haemofiltration procedures were used as proxy for acute kidney replacement therapy (KRT) in a critical care setting. Temporal trends in usage of AKI-specific ICD-10 codes were examined. A total of 356 million inpatient episodes of care over 22 years were analysed. Between 1998/1999 and 2019/2020, primary AKI diagnoses increased from 1.2 to 5.3 per thousand finished consultant episodes. From 2012/2013, when first recorded, secondary diagnoses doubled from 22.8 to 43.2 per thousand finished consultant episodes. The rate of growth in AKI was significantly greater than that of dementia, but not pneumonia or heart failure. The proportion of acute KRT procedures to total AKI diagnoses decreased. This could suggest that the substantial increase in AKI cases observed within NHS in the last two decades is a result of improved detection and coding, particularly of mild cases. In 2019/2020, 96\% of AKI cases were encoded as N17.9 (Acute renal failure, unspecified), despite 11 other codes being available. We believe that adoption of clinically orientated coding could facilitate further improvements in detection with benefits for clinical practice and research.
\end{abstract}

Keywords Nephrology $\cdot$ Acute kidney injury $\cdot$ Hospital Episode Statistics $\cdot$ National Health Service

\section{Introduction}

The last two decades have seen a growing recognition of acute kidney injury (AKI) as a major cause of morbidity and mortality in secondary care. This has been facilitated by the publication of the consensus criteria for AKI diagnosis in 2004 [1], followed by a number of interventions aiming to raise public and professional awareness and to improve AKI detection and outcomes. In the United Kingdom (UK), AKI

This article is part of the Topical Collection on Medicine

Zuzanna Bien

zuzanna.bien@nhs.net

1 Adult Critical Care Unit, The Royal London Hospital, Barts Health NHS Trust, Whitechapel Road, London E1 1FR, UK

2 Critical Care and Perioperative Medicine Research Group, William Harvey Research Institute, Queen Mary University of London, London, UK is estimated to account for up to 100,000 deaths each year, nearly a quarter of them thought to be preventable $[1,2]$.

The growing awareness of AKI as a serious and partially avoidable disease prompted healthcare policy decisions aimed at improving detection and treatment of the disease. The Think Kidneys campaign, which commenced in 2014, focused on educating the public and clinicians on risks and correct management of AKI [3]. In the same year, NHS England issued a Patient Safety Alert, requiring care providers around the country to unify the method of detection of AKI according to biochemical results [4]. It also mandated the issue of electronic alerts to clinicians when their patients' blood test results met the definition of AKI based on the 2012 KDIGO (Kidney Disease: Improving Global Outcomes) criteria [5]. Similar systems have been introduced worldwide [6] Many care providers paired the electronic alerts with "AKI bundles"-electronic checklists of investigations and interventions recommended for patients with an AKI [7]. The UK National Commissioning for Quality and Innovation (CQUIN) framework in 2015/2016 
selected AKI as one of the priority areas for improvement and attached financial incentives for care providers to improve their outcomes [8].

In the same time frame, the reported incidence of AKI has been on the rise both in the UK and worldwide. In a large US database, the number of records associated with AKI rose from $0.4 \%$ in 1998 to $2.1 \%$ in 2002 [9]; another American study estimated an annual growth in AKI cases at $11 \%$, with an average of 23.8 per thousand records being associated with an AKI between 1992 and 2001 [10]. It is unclear whether this rise reflects a true increase in incidence, or an improvement in AKI recognition and coding prompted by campaigns and financial incentives such as the ones described above. A large study looking at creatinine values, rather than clinical codes, found a stable incidence of around 5\% between 2006 and 2014, favouring the latter interpretation [11].

The last report on clinical coding of AKI within the NHS was published before the introduction of the Think Kidneys campaign, covering a period between 1998/1999 and 2012/2013. During that time, the authors reported a 12-fold increase in codes for AKI not requiring dialysis [12], and a 13-fold increase in codes for dialysed AKI [13]. Our study encompasses an extended period from 1998/1999 to 2019/2020, aiming to capture any change in AKI coding which could have arisen as a result of national campaigns and financial incentives. In our study, we differentiate between AKI coded as primary diagnosis (the main reason for admission), and that coded as a secondary diagnosis (appearing amongst up to 20 auxiliary codes for diagnoses that arose at any point during the hospital episode). We postulate that disproportionate rise in AKI coded as secondary diagnosis versus primary diagnosis could indicate increased reporting of less clinically significant cases rather than a true increase in incidence. To further support this hypothesis, we compare AKI cases to acute renal replacement therapy (RRT) procedures, suggesting that a lower proportion of acute RRT to total AKI numbers may indicate increased coding of mild disease. We also contrast AKI with three other diseases of comparable incidence-pneumonia, heart failure, and dementia-in order to elucidate whether the trends observed are specific to AKI, or can be generalised to other diagnostic entities. Lastly, we examine how the patterns of usage of individual AKI-related ICD-10 codes changed over time.

\section{Methods}

\section{Study Design}

This time-trend ecological study used anonymized, publicly available national data published by NHS Digital available under an Open Government License v3.0 [14]. Datasets from HES admitted patient care (APC) records contain information about all episodes of inpatient treatment in NHS hospitals in England. In HES Data Dictionary, a finished consultant episode (FCE) is defined as a continuous period under the care of a single consultant; one admission spell can contain multiple FCE. In contrast, a finished admission episode (FAE) represents the first episode in an admission spell [15]. Notes from all episodes of care are retrospectively reviewed by professional clinical coders, who assign diagnostic and procedural codes to each of them for reimbursement purposes [16]. Data since 1998 was downloaded to encompass all admissions between 1 April 1998 and 31 March 2020. A pre-specified analysis plan was followed [17].

\section{Diagnoses}

HES APC consists of diagnosis tables classified by the International Statistical Classification of Diseases and Related Health Problems, $10^{\text {th }}$ Revision [18]. (ICD-10) codes. Each finished consultant episode is associated with one primary diagnosis and either 7 (1998-2002), 14 (2002-2007), or 20 (2007-present) secondary diagnoses. For each ICD-10 code, an aggregate count is presented of the number of episodes where it featured as the primary diagnosis and amongst all diagnoses. Primary diagnosis database has been published since 1998/1999 and provides finished consultant episode and admission episode counts, whilst data on all diagnoses has been published since 2012/2013 and for consultant episodes only. Episodes are further subdivided by age into groups of 0-14 years, 14-59 years, 60-75 years, and 75 + up to 2011/2012; and into 24 smaller age brackets thereafter. A detailed description of the dataset is provided by Herbert et al. [19].

\section{Procedures and Interventions}

HES APC also includes procedure tables which are classified using the Office of Population Censuses and Surveys Classification of Interventions and Procedures, 4th revision (OPCS-4) classification [20]. Each episode has one primary procedure, defined as the most resource-intensive procedure performed, and either 12 (1998-2007) or 24 (2007-present) secondary procedures. For each OPCS-4 code, the table presents the number of times it featured as the primary procedure (further subdivided into emergency and elective); and amongst all procedures. 


\section{Clinical codes}

\section{ICD-10 Codes}

For identification of AKI cases, we used the ICD-10 classification proposed by Mansfield et al. [21]. Four digit ICD-10 codes were used for greater specificity (Table 1). HES database was pre-screened to identify the incidence of diagnoses of six additional disease entities: ischaemic heart disease, liver disease, urinary tract infection, pneumonia, dementia, and heart failure. The three diseases with population incidence most similar to AKI in 2012/2013 - pneumonia, dementia, and heart failure-were chosen as comparator diagnoses. Supplementary Table 1 contains a detailed list of code mappings used.

\section{OPCS-4 Codes for Acute Kidney Replacement Therapy (KRT)}

The OPCS-4 code X40.4 (haemofiltration) was included in the analysis. Haemofiltration is the predominant mode of KRT used in the intensive care unit (ICU) setting in the UK [22] and as such, it was chosen as a proxy measure for the number of cases of severe AKI requiring KRT.

\section{Software}

Microsoft Excel was used for data storage and initial processing [23]. R [24] was used for analysis and graphic data representation.

\section{Processing}

In certain categories, values in cells with fewer than 5 (or 7 , depending on the year) episodes were replaced with an

Table 1 ICD-10 codes used to represent AKI in the HES database, after Mansfield et al. [20]

\begin{tabular}{ll}
\hline ICD-10 & Description \\
\hline N14.0 & $\begin{array}{l}\text { Analgesic nephropathy } \\
\text { Nephropathy induced by other drugs, medica- } \\
\text { ments, and biological substances } \\
\text { Nephropathy induced by unspecified drug, } \\
\text { medicament, or biological substance }\end{array}$ \\
N14.2 & Acute renal failure with tubular necrosis \\
N17.0 & Acute renal failure with acute cortical necrosis \\
N17.1 & Acute renal failure with medullary necrosis \\
N17.2 & Other acute renal failure \\
N17.8 & Acute renal failure, unspecified \\
N17.9 & Unspecified kidney failure \\
N19.X & Postprocedural renal failure \\
N99.0 & Anuria and oliguria \\
R34.X & Abnormal results of kidney function studies \\
R94.4 &
\end{tabular}

asterisk to protect patient confidentiality. For the purposes of this analysis, these missing values were replaced with the maximum possible value they could have taken, i.e. 5 or 7 .

For each ICD-10 or OPCS- 4 code, we extracted the number of episodes during which it was recorded as the primary diagnosis and amongst all diagnoses. The difference between the two totals was calculated to produce the number of times each code was used as a secondary diagnosis.

From 2012/2013 onwards, age groups were coalesced from 24 into 4 brackets ( $0-14$ years, $15-59$ years, 60-74 years, and over 75 years) to match the format in earlier datasets.

For calculating proportions, the total yearly number of finished consultant episodes or finished admission episodes was used as the denominator.

Percentage growth was calculated as $C=\left(x_{i+1}-x_{i}\right) / x_{i}$, where $x_{i}+\cdots+x_{n}$ are the sequential yearly values. Annual average growth rate (AAGR) was calculated as $\left(C_{1}+\cdots+C_{n}\right) / n$.

We used a simple linear regression model to estimate the slope of the increase in all episodes of AKI and pneumonia, heart failure, and dementia. The addition of an interaction term between diagnosis type and time in years was used to test for statistical difference between diagnoses in the rate of growth.

A sensitivity analysis of time- and age-related changes in primary and secondary diagnoses of AKI was conducted where AKI was defined by N17-group codes only.

\section{Results}

A total of 356,034,130 finished consultant episodes of inpatient care over 22 years were analysed.

From the inception of records in 1998/1999 until $2019 / 2020$, the proportion of all consultant episodes with a primary diagnosis of AKI increased from 1.2 to 5.3 per thousand (Fig. 1), whilst the absolute number of primary AKI diagnoses increased eightfold from 14,041 to 110,960 with an average annual growth rate (AAGR) of $10.5 \%$. In comparison, the proportion of all admission episodes with a primary diagnosis of AKI increased from 0.9 to 3.3 per thousand (or from 10,120 to 56,266 in absolute terms) with an AAGR of $8.6 \%$.

From 2012/2013, when they were first recorded, until $2019 / 2020$, the proportion of consultant episodes with a secondary diagnosis of AKI increased from 22.8 to 43.2 per thousand, and the absolute number grew more than twice from 404,019 to 902,671 with an AAGR of $12.4 \%$ (Table 2; extended version available in Supplementary Table 2). There was no subdivision into consultant and admission episodes in the secondary diagnosis data. 
Fig. 1 Finished consultant episodes (FCE) associated with a primary (left) and secondary (right) diagnosis of AKI by age, represented as number of diagnoses per thousand FCE in each age group. Data on secondary diagnoses is only available from $2012 / 2013$

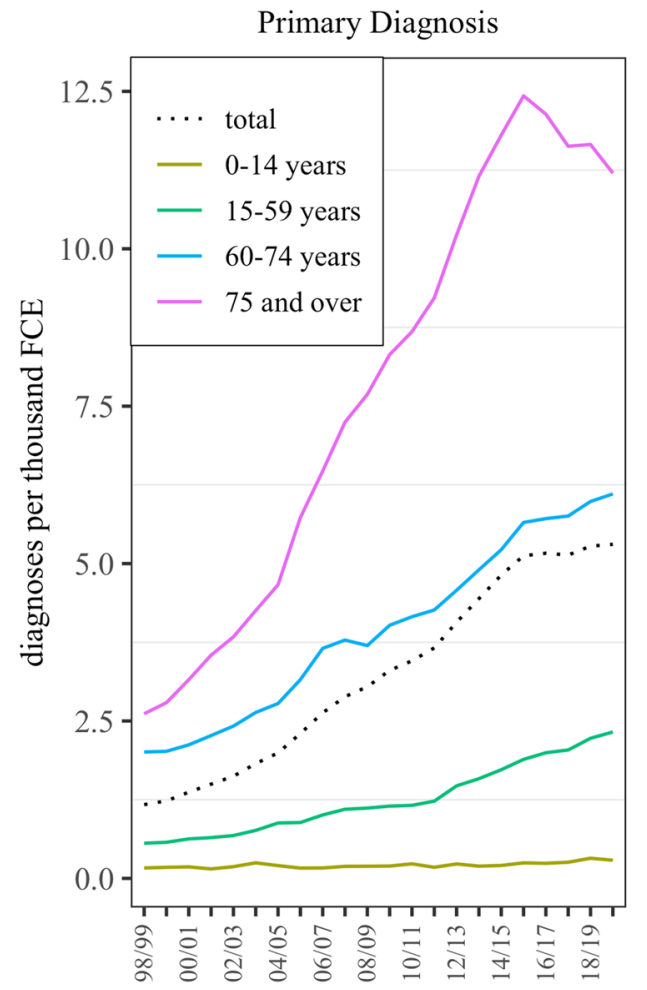

In total, there were $1,248,157$ finished consultant episodes (including 673,329 finished admission episodes) associated with a primary diagnosis of AKI between 1998/1999 and 2019/2020 and 5,581,147 finished consultant episodes associated with a secondary diagnosis of AKI between 2012/2013 and 2019/2020.

\section{Age Breakdown}

Of all age groups, $75+$ were most likely to have a diagnosis of AKI associated with a finished consultant episode (Fig. 1). Between 1998/1999 and 2019/2020, this age group also experienced the steepest rise in the number of AKI diagnoses made: primary diagnoses increased from 2.6 to 11.2 per thousand consultant episodes; and secondary diagnoses from 61.3 to 95.8 per thousand consultant episodes (Fig. 1). The proportion of consultant episodes associated with an AKI, as well as the rate of growth, was less pronounced as the age diminished (Supplementary Table 3).

In 1998/1999, 41.4\% of primary diagnoses of AKI were made in the $\geq 75$ age group. In 2019/2020, this proportion grew to 55.5\% . Between 2012/2013 and 2019/2020, the proportion of secondary diagnoses accounted for by the $\geq 75$ age group diminished from $64.2 \%$ to $58.3 \%$ (Supplementary Fig. 1). This proportion is significantly greater than the percentage of general hospital admission recorded in this age group. In 1998/1999, $18.5 \%$ of the general hospital population was $\geq 75$, increasing to $23.9 \%$ in $2012 / 2013$ and $26.3 \%$ in 2019/2020. The mean age of a hospital inpatient in that time increased from 45 to 54 years (Supplementary Table 4).

\section{AKI vs. Diseases of Similar Incidence}

Total number of consultant episodes with primary or secondary AKI diagnosis increased from 476,113 to 1,013,631 between 2012/2013 and 2019/2020 with an AAGR of 11.6\%. In comparison, the number associated with a diagnosis of pneumonia rose from 594,315 to $1,041,452$ (AAGR 8.5\%); heart failure from 650,434 to $1,271,934$ (AAGR 10.1\%); and dementia from 478,668 to 868,907 (AAGR 8.9\%) (Supplementary Table 5). In this time frame, the proportion of all consultant episodes associated with a diagnosis of AKI grew from 26.9 to 48.5 episodes per thousand, compared with an increase from 33.5 to 49.8 in pneumonia, 26.7 to 60.8 in heart failure, and 27.0 to 41.6 in pneumonia cases per thousand consultant episodes (Fig. 2). The number of diagnoses increased significantly between 2012/2013 and 2019/2020 for all of the diseases (all $p<0.001$ ). The rate of growth of AKI was significantly greater than that of dementia $(p=0.02)$ but not that of heart failure $(p=0.71)$ or pneumonia $(p=0.16)$.

\section{Acute KRT}

The total number of finished consultant episodes associated with a haemofiltration code increased from 15,429 in 

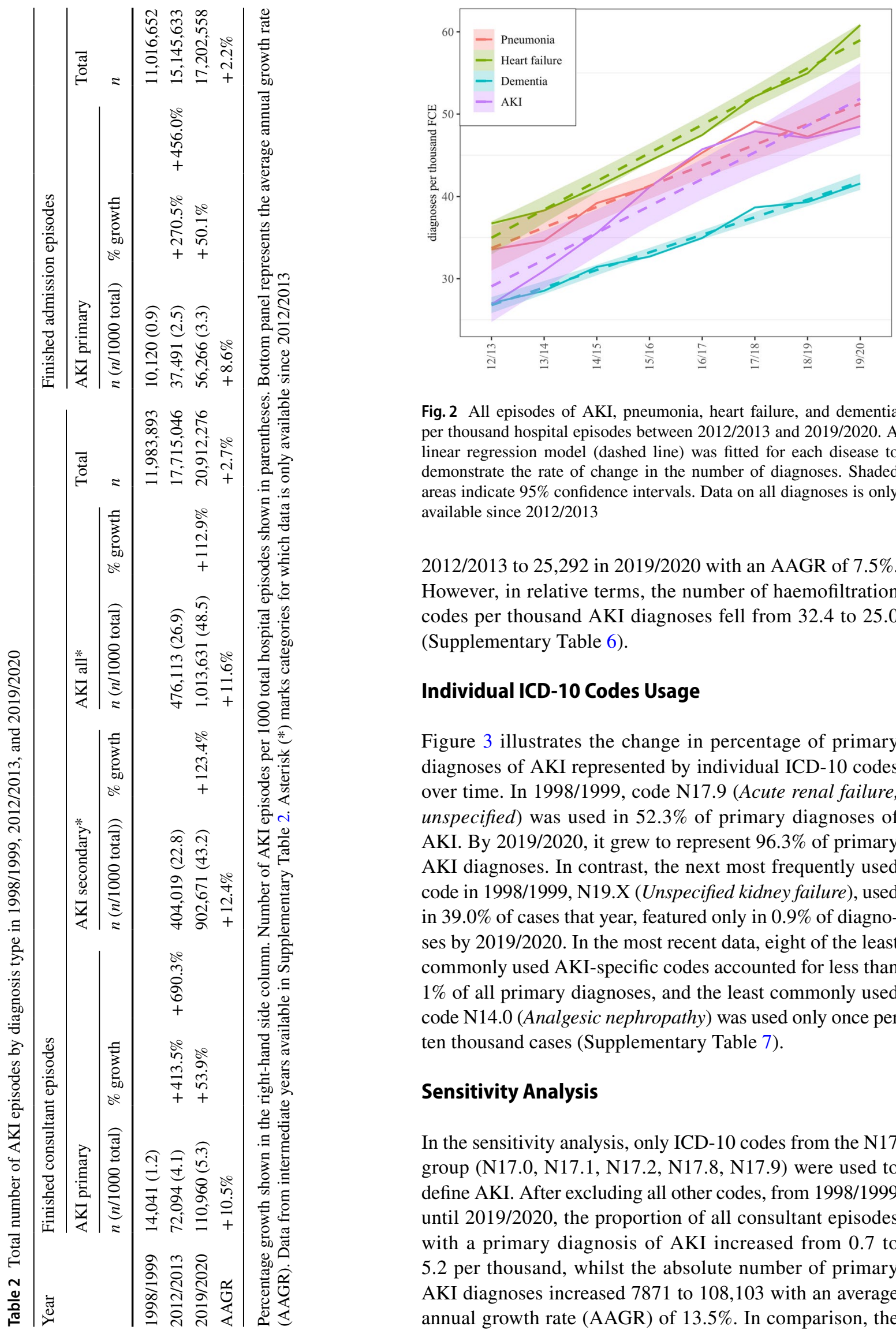

Fig. 2 All episodes of AKI, pneumonia, heart failure, and dementia per thousand hospital episodes between 2012/2013 and 2019/2020. A linear regression model (dashed line) was fitted for each disease to demonstrate the rate of change in the number of diagnoses. Shaded areas indicate $95 \%$ confidence intervals. Data on all diagnoses is only available since $2012 / 2013$

2012/2013 to 25,292 in 2019/2020 with an AAGR of 7.5\%. However, in relative terms, the number of haemofiltration codes per thousand AKI diagnoses fell from 32.4 to 25.0 (Supplementary Table 6).

\section{Individual ICD-10 Codes Usage}

Figure 3 illustrates the change in percentage of primary diagnoses of AKI represented by individual ICD-10 codes over time. In 1998/1999, code N17.9 (Acute renal failure, unspecified) was used in $52.3 \%$ of primary diagnoses of AKI. By 2019/2020, it grew to represent $96.3 \%$ of primary AKI diagnoses. In contrast, the next most frequently used code in 1998/1999, N19.X (Unspecified kidney failure), used in $39.0 \%$ of cases that year, featured only in $0.9 \%$ of diagnoses by 2019/2020. In the most recent data, eight of the least commonly used AKI-specific codes accounted for less than $1 \%$ of all primary diagnoses, and the least commonly used code N14.0 (Analgesic nephropathy) was used only once per ten thousand cases (Supplementary Table 7).

\section{Sensitivity Analysis}

In the sensitivity analysis, only ICD-10 codes from the N17 group (N17.0, N17.1, N17.2, N17.8, N17.9) were used to define AKI. After excluding all other codes, from 1998/1999 until 2019/2020, the proportion of all consultant episodes with a primary diagnosis of AKI increased from 0.7 to 5.2 per thousand, whilst the absolute number of primary AKI diagnoses increased 7871 to 108,103 with an average annual growth rate (AAGR) of $13.5 \%$. In comparison, the 
Fig. 3 Percentage of all AKI episodes by individual ICD-10 codes. The legend is arranged in a descending order of frequency based on data from 1998/1999.

The left-hand side panel presents a magnified portion of the full graph to allow discrimination of the less frequent categories

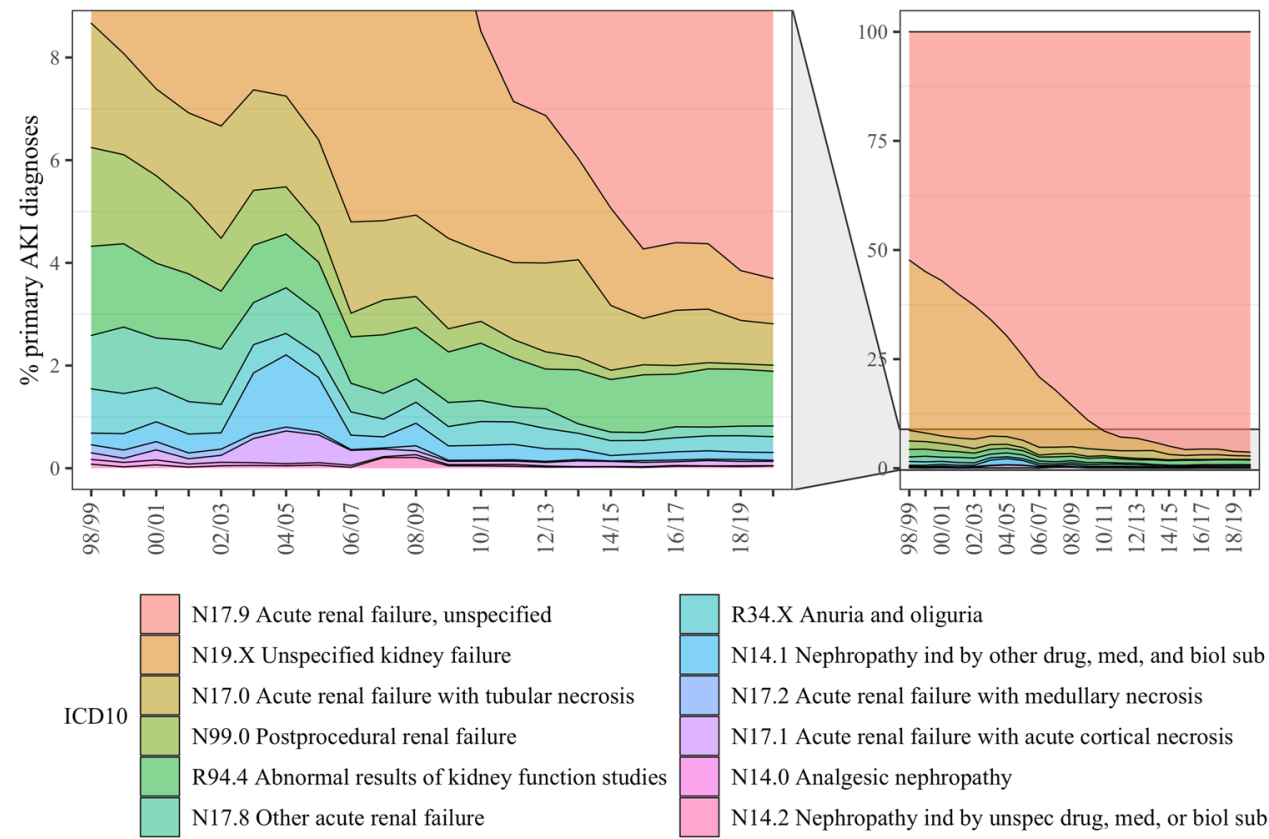

proportion of all admission episodes with a primary diagnosis of AKI increased from 0.5 to 3.1 per thousand (or from 5271 to 53,938 in absolute terms) with an AAGR of $11.9 \%$ (Supplementary Table 8).

From 2012/2013, when they were first recorded, until $2019 / 2020$, the proportion of consultant episodes with a secondary diagnosis of AKI increased from 20.0 to 41.3 per thousand, and the absolute number grew more than twice from 354,761 to 864,433 with an AAGR of $13.9 \%$ (Supplementary Table 8). As before, there was no subdivision into consultant and admission episodes in the secondary diagnosis data.

\section{Discussion}

Over the last two decades, the proportion of finished consultant episodes of inpatient care with AKI as the primary diagnosis has grown more than four-fold. Since they were first recorded 7 years ago, the proportion of consultant episodes with AKI as a secondary diagnosis has nearly doubled. In the same time frame, the number of acute KRT procedures diminished in relation to the total number of AKI diagnoses. In 2019/2020, more than half of AKI diagnoses were made in those aged 75 and over; in this age group, one in ten of all episodes of care was associated with an AKI.

An earlier American study of 5 million Medicare beneficiaries between 1992 and 2001 using ICD-9 codes observed an annual growth rate of $11 \%$ and an average incidence of 23.8 cases per thousand discharges [10]. We observed a comparable growth rate of $11.6 \%$; however, AKI diagnoses were associated with a more significant proportion of all hospital episodes, growing from 26.9 to 48.5 per thousand between 2012/2013 and 2019/2020. It remains unclear whether the rapid rise observed reflects a true rise in incidence of AKI, or improved detection and coding.

Increasing age and burden of co-morbid diseases of the hospital population could certainly contribute to a rise in incidence. In this study, the increase in the number of AKI cases has been driven primarily by those aged 75 and over. This age group accounts for a growing proportion of the hospital population, from a fifth in 1998/1999 to a quarter in 2019/2020 (compared with 7\% in 1998 and $8 \%$ in 2018 of the general population) [25]. The elderly have multiple susceptibilities to developing AKI including the presence of co-morbid disease (in particular hypertension, diabetes, and heart failure), poorer baseline renal function and renal reserve, and polypharmacy [26]. The other diseases selected as comparators in this study - pneumonia, heart failure, and dementia_-all disproportionately affect the elderly population, and all have grown to account for a greater proportion of all episodes of care in the last 7 years, suggesting that the nation-wide disease profile might be changing to encompass a growing proportion of diseases of old age. Amongst patients of all ages, increasing population prevalence of predisposing conditions such as diabetes, obesity, and chronic kidney disease is also likely contributing to the growth in AKI [27].

Despite this, an American study of hospital inpatients that evaluated biochemical data (rather than clinical codes) over a 9-year period found that the incidence of AKI did not increase [11]. This could suggest that the rapid rise in cases observed in this study, and reported elsewhere, could be attributed to improved detection rather than true increase 
in incidence. We found that the average annual growth in AKI as secondary diagnosis was greater than that of primary cases, in particular between 2012/2013 and 2016/2017, when many campaigns centred around raising awareness of AKI were taking place. Increased awareness may have led to improved coding of milder cases of AKI which were not the main reason for hospitalisation, but arose secondarily to another diagnosis (and later in the course of the admission spell, as suggested by lower prevalence of AKI amongst initial admission episodes compared to all episodes of care). In particular, the introduction of automatic electronic alerts likely led to AKI diagnoses being automatically added to an episode, even if not clinically significant. Favouring this interpretation, we found that the number of patients receiving haemofiltration in the critical care setting relative to number of AKI episodes has fallen steadily from 2012/2013 until present, suggesting that a growing proportion of AKI diagnoses is accounted for by mild cases not requiring renal replacement. An increasing recognition of clinically insignificant cases could explain why studies of e-alerts and AKI bundles have not reliably found a positive impact on patient outcomes [28, 29].

Notably, even at its peak in 2019/2020, only one in twenty hospital episodes included in this study was associated with an AKI. In comparison, studies using biochemical parameters estimated the incidence of AKI in hospitalised patients to be $5-16 \%[11,30]$; some quoted rates as high as $23 \%$ [31, 32]. One Scottish study of two major hospitals between 2013 and 2017 found that only between 25.7 and $53.8 \%$ of biochemically defined cases of AKI were appropriately coded depending on severity and location (although this study used the N17 group codes only and therefore may have missed a proportion of cases) [33]. In this context, our study suggests that despite the rapid growth in the number of AKI diagnoses, many cases may continue to be missed from records. This conclusion is supported by the UK Renal Registry report [34], which linked HES records with biochemical data to demonstrate that only between $\sim 45$ and $80 \%$ of inpatient episodes where AKI occurred are given the appropriate ICD-10 code, depending on AKI stage (accuracy improves with increasing severity of disease).

One barrier to understanding the epidemiology of AKI may be the lack of specificity of ICD-10 codes being used. We found that the practice of clinical coding for AKI has changed significantly in the last two decades. From over half in 1998/1999, code N17.9 (Acute renal failure, unspecified) has grown to account for nearly all AKI episodes in 2019/2020. Out of 12 codes available, the bottom eight jointly accounted for less than $1 \%$ of all diagnoses. Codes such as R94.4 (Abnormal results of kidney function studies) and N19.X (Unspecified kidney failure) are virtually synonymous with N17.9 and do not provide much additional diagnostic value. ICD-10 follows a system of classification of AKI based primarily on pathophysiology. In practice, the majority of cases of AKI are multi-factorial, and most never receive a tissue diagnosis [35]. Adoption of more clinically relevant coding system for AKI, more in line with KDIGO criteria and electronic warning systems, such as that offered by SNOMED-CT [36], would distinguish between episodes based on the degree of severity and could prove more informative in guiding treatment and research.

This study has a number of strengths. It includes the whole population of hospitalised patients in England in the last 22 years and some 356 million episodes of care. Data collection on such scale is only possible in a universal healthcare system such as the NHS and vastly improves generalisability of the findings. Whilst other studies have examined the validity of ICD-10 codes for detecting biochemical cases of AKI [37-39], to our knowledge, none to date has examined the trends in usage of different ICD-10 entities over the years, which provides an interesting background for future discussions regarding the validity and clinical utility of this classification. We analysed the trends in age of the hospital population as well as data on other diseases which allowed us to place our findings in context.

However, there are also some limitations. Some of the ICD-10 codes used in the primary analysis may be poorly specific to AKI, leading to overestimation of the number of cases. In order to control for this, we conducted a sensitivity analysis using N17-group codes only. This revealed broadly similar temporal and age-related trends, although the average annual growth rate of AKI coded as primary diagnosis was greater than in the primary analysis where non-N17 codes were also included. This is likely related to the changing habits of code usage over the years, with growing propensity to use N17 codes in more recent times.

Due to the nature of OPCS-4 codes, we were only able to distinguish between different modes, not indications for KRT. Haemofiltration is the predominant mode of KRT used in the intensive care unit (ICU) setting in the UK [22] and as such, it was chosen as a proxy measure for the number of cases of severe AKI requiring KRT. However, without data on individual indications, some non-AKI cases needing haemofiltration may have been included. Additionally, some patients with AKI who are treated with haemodialysis outside of ICU may have been missed [40]. Using a recordlevel analysis of the HES database (where data regarding individual episodes can be accessed), Kolhe and colleagues estimated the number of cases of AKI requiring dialysis to be 11,164 in 2012/2013; indicating that our method, which yielded 15,429 cases that year, might have led to an overestimation [13].

Some other limitations can be identified. The HES database contains aggregate data only; cases cannot be traced back to individual patients, for example to identify patients with an AKI code who also required KRT. The ICD-10 
classification system has been shown to have poor sensitivity in identifying biochemical AKI cases [39]. Data regarding secondary diagnoses and procedures has only been published since 2012/2013; therefore, we could only analyse the trends in these variables in the last 8 years. The other diseases which we used to contextualise our findings may not be independent from AKI; in particular pneumonia and heart failure can both be precipitating factors. Lastly, the study might have limited external validity for other healthcare systems outside of England.

\section{Conclusion}

The number of AKI diagnoses has grown significantly in the last two decades. Most of the cases affect the elderly population. Although the classification system does not allow us to distinguish the severity of cases, the relative decrease in haemofiltration procedures in proportion to AKI diagnoses, as well as greater increase in secondary than primary diagnoses, could suggest that it is primarily due to a growth in recognition of mild cases. Despite this surge, the number of diagnoses coded remains lower than the prevalence of AKI estimated by studies based on biochemical results. The lack of clarity and simplicity of ICD-10 coding system might contribute to sub-optimal reporting. Adoption of more clinically significant codes, such as SNOMED-CT, which distinguish between degrees of severity of AKI, could facilitate earlier and more targeted treatment, simplify coding, and facilitate research.

Supplementary Information The online version contains supplementary material available at https://doi.org/10.1007/s42399-022-01127-y.

Author Contribution Study concept and design: JRP, YIW, AJF. Study protocol and analysis plan: ZB, JRP, YIW. Data analysis: ZB, AJF, AJR, YIW. Critical review of finding: ZB, RMP, JRP, YIW. Manuscript writing: ZB, JRP, YIW. Review of final submission: all authors

Code Availability $\mathrm{R}$ code for the analysis is available on request.

\section{Declarations}

Ethics Approval This study used anonymized, publicly available national data published by NHS Digital available under an Open Government License v3.0 and as such it did not require an ethics committee approval [14].

Consent to Participate Not applicable to this study.

Consent for Publication All relevant parties consent to the publication of this study.

Conflict of Interest Dr John Prowle has received consultancy fees from MediBeacon, Nikkiso Europe $\mathrm{GmbH}$, and Quark Pharmaceuticals; speaker honoraria from Baxter, Fresenius Medical Care, and Nikkiso
Europe $\mathrm{GmbH}$; and research support from bioMérieux. The other authors have declared no competing interests.

Data Transparency The data used in the study is published by NHS Digital and is publicly available for access.

Data Sharing The data used in the study is published by NHS Digital and is publicly available for access.

Open Access This article is licensed under a Creative Commons Attribution 4.0 International License, which permits use, sharing, adaptation, distribution and reproduction in any medium or format, as long as you give appropriate credit to the original author(s) and the source, provide a link to the Creative Commons licence, and indicate if changes were made. The images or other third party material in this article are included in the article's Creative Commons licence, unless indicated otherwise in a credit line to the material. If material is not included in the article's Creative Commons licence and your intended use is not permitted by statutory regulation or exceeds the permitted use, you will need to obtain permission directly from the copyright holder. To view a copy of this licence, visit http://creativecommons.org/licenses/by/4.0/.

\section{References}

1. Bellomo R, Ronco C, Kellum JA, Mehta RL, Palevsky P. Acute renal failure - definition, outcome measures, animal models, fluid therapy and information technology needs: the Second International Consensus Conference of the Acute Dialysis Quality Initiative (ADQI) Group. Crit Care. 2004;8(4):R204-12.

2. Stewart J, Smith N, Kelly K, Mason M, Findlay G. Adding insult to injury. A review of patients who died in hospital with a primary diagnosis of acute kidney injury (NCEPOD). [Internet]. 2009 [accessed 2020 Oct 30] p. 1-100. Available from: http:// www.ncepod.org.uk/2009aki.html.

3. Think Kidneys. Think Kidneys National AKI programme: review and evaluation report [Internet]. February 2017 [accessed 2020 Oct 30]. p. 1-52. Available from: https://www. thinkkidneys.nhs.uk/aki/wp-content/uploads/sites/2/2017/02/ NHS-Think-Kidneys-AKI-Programme-Review-and-Evaluation. pdf.

4. Selby NM, Hill R, Fluck RJ, on behalf of the NHS England. Think Kidneys AKI programme. Standardizing the early identification of acute kidney injury: the NHS England National Patient Safety Alert. Nephron. 2015;131(2):113-7.

5. Kellum JA, Lameire N, Aspelin P, et al. Kidney Disease: Improving Global Outcomes (KDIGO) Acute Kidney Injury Work Group. KDIGO clinical practice guideline for acute kidney injury. Kidney Int Suppl. 2012;2(1):1-38.

6. Lachance P, Villeneuve P-M, Rewa OG, et al. Association between e-alert implementation for detection of acute kidney injury and outcomes: a systematic review. Nephrol Dial Transplant. 2017;32(2):265-72.

7. Bagshaw SM. Acute kidney injury care bundles. Nephron. 2015;131(4):247-51.

8. NHS England Contracting and Incentives Team. Commissioning for Quality and Innovation (CQUIN). guidance for 2015/16. [Internet]. 2015 Mar [accessed 2020 Nov 6]. Available from: https://www.england.nhs.uk/wp-content/uploads/2015/03/9cquin-guid-2015-16.pdf.

9. Waikar SS, Curhan GC, Wald R, McCarthy EP, Chertow GM. Declining mortality in patients with acute renal failure, 1988 to 2002. J Am Soc Nephrol. 2006;17(4):1143-50. 
10. Xue JL, Daniels F, Star RA, et al. Incidence and mortality of acute renal failure in Medicare beneficiaries, 1992 to 2001. J Am Soc Nephrol. 2006;17(4):1135-42.

11. Kashani K, Shao M, Li G, et al. No increase in the incidence of acute kidney injury in a population-based annual temporal trends epidemiology study. Kidney Int. 2017;92(3):721-8.

12. Kolhe NV, Muirhead AW, Wilkes SR, Fluck RJ, Taal MW. The epidemiology of hospitalised acute kidney injury not requiring dialysis in England from 1998 to 2013: retrospective analysis of Hospital Episode Statistics. Int J Clin Pract. 2016;70(4):330-9.

13. Kolhe NV, Muirhead AW, Wilkes SR, Fluck RJ, Taal MW. National trends in acute kidney injury requiring dialysis in England between 1998 and 2013. Kidney Int. 2015;88(5):1161-9.

14. Open Government Licence version 3.0. [Internet; accessed 2020 Sep 9] Available from: http://www.nationalarchives.gov.uk/doc/ open-government-licence/version/3/.

15. NHS Digital. HES Data Dictionary: Hospital Admitted Patient Care Activity. [Internet; accessed 2021 Mar 14]. Available from: https://digital.nhs.uk/data-and-information/publications/statisti$\mathrm{cal} /$ hospital-admitted-patient-care-activity.

16. Data Quality Statement. NHS Digital. [Internet; accessed 2021 Dec 28]. Available from: https://digital.nhs.uk/data-and-infor mation/publications/statistical/hospital-admitted-patient-careactivity/2019-20/data-quality-statement.

17. Bien Z, Fowler AJ, Robbins AJ, Prowle JR, Pearse RM, Wan YI. Trends in hospital admissions associated with an acute kidney injury in England 1998-2020: statistical analysis proposal. [Internet; accessed 2020 Dec 6]. Available from: https://www.qmul. ac.uk/ccpmg/media/critical-care-and-pmg/documents/HES-AKISAP.pdf.

18. ICD-10: International statistical classification of diseases and related health problems. Geneva: World Health Organization. [Internet] 2019 [accessed 2020 Nov 17]. Available from: https:// icd.who.int/browse10/2019/en.

19. Herbert A, Wijlaars L, Zylbersztejn A, Cromwell D, Hardelid P. Data resource profile: Hospital Episode Statistics Amitted Patient Care (HES APC). Int J Epidemiol. 2017;46(4):1093-1093i.

20. NHS Digital. National clinical coding standards OPCS-4: accurate data for quality information. [Internet] 2018 [accessed $2020 \mathrm{Nov}$ 17]. Available from https://hscic.kahootz.com/gf2.ti/f/762498/ 46448197.2/PDF/-/National_Coding_Standards_OPCS4_refer ence_book_2019.pdf.

21. Mansfield KE, Nitsch D, Smeeth L, Bhaskaran K, Tomlinson LA. Prescription of renin-angiotensin system blockers and risk of acute kidney injury: a population-based cohort study. BMJ Open. 2016;6(12):e012690.

22. Wright SE, Bodenham A, Short AIK, Turney JH. The provision and practice of renal replacement therapy on adult intensive care units in the United Kingdom. Anaesthesia. 2003;58(11):1063-9.

23. Microsoft Corporation. Microsoft Excel. [Internet, accessed 2020 Nov 6]. 2020 (version 16.43). Available from: https://office.micro soft.com/excel.

24. R Core Team. R: a language and environment for statistical computing. [Internet, accessed 2020 Nov 6]. Vienna, Austria.: R Foundation for Statistical Computing.; 2019. Available from: https:// www.R-project.org/.

25. Office for National Statistics. UK population pyramid interactive [Internet]. [accessed 2020 Aug 20]. Available from: https://www. ons.gov.uk/peoplepopulationandcommunity/populationandmigrati on/populationestimates/articles/ukpopulationpyramidinteractive/ 2020-01-08.

26. Chao C-T, Tsai H-B, Lin Y-F, Ko W-J. Acute kidney injury in the elderly: only the tip of the iceberg. J Clin Gerontol Geriatr. 2014;5(1):7-12.

27. Siew ED, Davenport A. The growth of acute kidney injury: a rising tide or just closer attention to detail? Kidney Int. 2015;87(1):46-61.

28. Kashani KB. Automated acute kidney injury alerts. Kidney Int. 2018;94(3):484-90.

29. Wilson FP, Shashaty M, Testani J, Aqeel I, Borovskiy Y, Ellenberg SS, et al. Automated, electronic alerts for acute kidney injury: a single-blind, parallel-group, randomised controlled trial. Lancet. 2015;385(9981):1966-74.

30. Zeng X, McMahon GM, Brunelli SM, Bates DW, Waikar SS. Incidence, outcomes, and comparisons across definitions of AKI in hospitalized individuals. Clin J Am Soc Nephrol. 2014;9(1):12-20.

31. Susantitaphong P, Cruz DN, Cerda J, Abulfaraj M, Alqahtani F, Koulouridis I, et al. World incidence of AKI: a meta-analysis. Clin J Am Soc Nephrol. 2013;8(9):1482-93.

32. Wang HE, Muntner P, Chertow GM, Warnock DG. Acute kidney injury and mortality in hospitalized patients. Am J Nephrol. 2012;35(4):349-55.

33. Logan R, Davey P, De Souza N, Baird D, Guthrie B, Bell S. Assessing the accuracy of ICD-10 coding for measuring rates of and mortality from acute kidney injury and the impact of electronic alerts: an observational cohort study. Clin Kidney J. 2019;19(1):1-8.

34. UK Renal Registry (UKRR). Acute kidney injury (AKI) in England - a report on the nationwide collection of AKI warning test scores from 2018. [Internet; accessed 2020 Jan 3]. Available from: https://ukkidney.org/sites/renal.org/files/AKI_report_FINAL_ 13072020.pdf.

35. Waikar SS, McMahon GM. Expanding the role for kidney biopsies in acute kidney injury. Semin Nephrol. 2018;38(1):12-20.

36. SNOMED-CT. [Internet; accessed 2020 Nov 6]. Available from: https://www.snomed.org/.

37. Tomlinson LA, Riding AM, Payne RA, Abel GA, Tomson CR, Wilkinson IB, et al. The accuracy of diagnostic coding for acute kidney injury in England - a single centre study. BMC Nephrol. 2013;14(1):58.

38. Grams ME, Waikar SS, MacMahon B, Whelton S, Ballew SH, Coresh J. Performance and limitations of administrative data in the identification of AKI. Clin J Am Soc Nephrol. 2014;9(4):682-9.

39. Logan R, Baird D, Davey P, Desouza N, Guthrie B, Bell S. ICD10 coding - an unreliable indicator for acute kidney injury rates and outcomes in hospital in-patients. Nephrol Dial Transplant. 2019;1(34):34.

40. Palevsky PM. Renal replacement therapy in acute kidney injury. Adv Chronic Kidney Dis. 2013;20(1):76-84.

Publisher's Note Springer Nature remains neutral with regard to jurisdictional claims in published maps and institutional affiliations. 